\title{
Análise do déficit e da concentração de oxigênio dissolvido no rio Piranhas-Açu, sujeito a lançamento de efluentes, mediante a utilização do modelo de Streeter-Phelps simplificado
}

\author{
The analysis of deficit and the concentration of dissolved oxygen in \\ the Piranhas-Açu river as likely to receive the launching of effluents \\ by means of the simplified Streeter-Phelps model
}

\section{Victor Prisco Diogo de Holanda \\ victor_prisco@hotmail.com Universidade de Fortaleza}

Thainá Rayanne Soares thainasoares90@gmail.com Universidade de Fortaleza

Roani Simões Veras roaniveras30@gmail.com Universidade de Fortaleza

Ingrid Fernandes de Oliveira Alencar ingrid.foa@hotmail.com Universidade de Fortaleza

Raquel Jucá de Moraes Sales raqueljuca@gmail.com Universidade de Fortaleza

\begin{abstract}
Resumo
Neste estudo, foi utilizado o modelo de Streeter-Phelps simplificado, que permite o cálculo dos parâmetros déficit de OD e o OD de forma simples no estudo de qualidade de água do rio PiranhasAçu, localizado no estado do Rio Grande do Norte. Para tanto, foram estabelecidos cenários de simulação a fim de avaliar a capacidade do rio em receber efluentes de fontes pontuais. Como resultado observou-se que quanto maior a concentração lançada no rio, menores serão as concentrações de OD, conforme previsto em literatura, evidenciando a veracidade do modelo. Verificou-se que, em períodos de estiagem, a capacidade de autodepuração do rio é pequena, elevando a presença de poluentes. Neste período, o rio não pode receber lançamentos maiores que $100 \mathrm{mg} / \mathrm{L}$. Contudo, em períodos de cheia, suporta concentrações maiores que $500 \mathrm{mg} / \mathrm{L}$. Este tipo de estudo facilita o estabelecimento de critérios de avaliação de qualidade de água em locais desprovidos de informação, uma vez que não necessita de muitos dados de entrada para um diagnóstico preliminar.
\end{abstract}

Palavras-chave: Oxigênio Dissolvido. Qualidade de água. Streeter-Phelps.

\begin{abstract}
In this study, we used the Streeter-Phelps simplified model that allows the calculation of OD deficit parameters and simply OD in the study of water quality of the river Piranhas-Açu (RN). For that purpose, simulation scenarios were established to assess the river's capacity for receiving wastewater from point sources. As a result, it has been observed that the higher the concentration released into the river is, the lower the DO concentrations appear, as provided in the literature, evidencing the accuracy of the model. It was found that, in periods of drought, the self-purification capacity of the river is small, raising the presence of river pollutants. During this period, the river should not receive more entries than $100 \mathrm{mg} / \mathrm{L}$. However, during rainy seasons, the river supports concentrations greater than $500 \mathrm{mg} / \mathrm{L}$. This type of study facilitates the establishment of criteria for evaluation of the quality of water in places devoid of information, since it does not require a lot of input data for a preliminary diagnosis.
\end{abstract}

Keywords: Dissolved Oxygen. Quality water. Streeter-Phelps.

\section{Considerações iniciais}

Os rios são a principal destinação dos lançamentos de esgotos brutos ou tratados (SPERLING, 2007). Devido a isso, considerando que água é essencial para os seres vivos, para a produção agrícola, para o uso doméstico e para os processos industriais (HU, 2009), é imprescindível a gestão e fiscalização de seu uso de forma que seja garantida a qualidade e a demanda desse bem. Atualmente, sérios problemas vêm sendo enfrentados devido à escassez de água e, principalmente, à redução de sua qualidade. A avaliação e o conhecimento da qualidade da água são de extrema importância para o adequado gerenciamento dos recursos hídricos, servindo, assim, como um indicador para o estágio de degradação ou conservação de um simples corpo d'água (ALVAREZ, 2010). 
De acordo com Sperling (2005), diversos parâmetros podem representar a qualidade da água, traduzindo suas principais características químicas, físicas e biológicas. O oxigênio dissolvido (OD) e a demanda bioquímica de oxigênio (DBO) são os principais parâmetros para estudos relacionados com a qualidade da água (VASCONCELLOS, 2013). Conforme Sanders (2009), a qualidade e a potabilidade da água estão diretamente relacionadas à oxigenação do corpo hídrico. O oxigênio é demandado biológica e quimicamente, oxidando os compostos tóxicos presentes nos corpos hídricos e diminuindo a toxidade dos mesmos. Segundo Sales (2014), quando tratamos de sistemas aquáticos, o consumo de oxigênio dissolvido na água, decorrente do processo de estabilização da matéria orgânica, é um dos principais fatores de poluição no meio ambiente.

Uma eficiente forma de se avaliar os impactos causados pelo lançamento de cargas poluidoras, bem como de analisar os cenários de intervenção e medidas de controle ambiental, é a utilização de modelos matemáticos de qualidade de águas (SPERLING, 2007). Entre os modelos matemáticos existentes, o pioneiro, e um dos mais utilizados até os dias de hoje, é o modelo de Streeter-Phelps. Desde então, o modelo tem sido generalizado por muitos pesquisadores, como Dobbins (1964), O'Connor (1967), Chapra (1997), e tantos outros. Devido à sua simplicidade e sua baixa necessidade de parâmetros e dados de entrada, esse modelo é bastante utilizado em nosso país para simulações de oxigênio dissolvido (SPERLING, 2007). Esse modelo foi desenvolvido por Harold Warner Streeter e Earle Bernard Phelps, e se baseia na hipótese de que a taxa de desoxigenação é proporcional à concentração da matéria orgânica presente em um determinado instante de tempo (STREETER, PHELPS, 1925).

A partir do modelo de Streeter-Phelps é possível analisar a capacidade de autodepuração de rios naturais, processo pelo qual a água de um corpo hídrico volta ao seu estado original, ou seja, se recupera de um estado de degradação sofrido, momento em que o equilíbrio é novamente alcançado no ecossistema (SALES, 2014). Neste estudo, utilizouse o modelo de Streeter-Phelps para verificar a capacidade do rio Piranhas-Açu, localizado no Rio Grande do Norte, em se recuperar mediante os processos de autodepuração, verificando a concentração de OD presente no rio após o lançamento de uma fonte pontual, analisado no cenário 1 em estudo. Foi verificada também a classificação do rio perante os parâmetros estabelecidos pela Resolução $n^{\circ} 357$ do CONAMA, analisada no cenário 2, bem como a concentração de OD nos períodos de cheia (entre fevereiro e maio) e de estiagem (entre agosto e novembro) no cenário 3.

Este tipo de estudo auxilia o estabelecimento de critérios de avaliação de qualidade de água e, consequentemente, a tomada de decisão por parte de gestores ambientais em locais carentes de informação, uma vez que não necessita de muitos dados de entrada para um diagnóstico preliminar.

\section{Metodologia}

Como aporte metodológico, a presente pesquisa trata da aplicação do modelo simplificado de Streeter-Phelps no estudo de qualidade das águas do rio Piranhas-Açu, bem como a capacidade de autodepuração do rio ao receber diferentes lançamentos, considerando apenas fontes pontuais. Neste estudo, foi utilizado o modelo na sua forma simplificada, com apenas os critérios e processos mais básicos envolvidos no processo de autodepuração de um corpo hídrico. O sucesso desta pesquisa poderá ajudar na verificação e aprovação de outorgas para o lançamento de efluentes em rios.

\subsection{Formulação do modelo de Streeter-Phelps simplificado}

As principais variáveis no modelo de Streeter-Phelps são a DBO, o déficit de OD e as concentrações de OD. Abaixo temos suas equações matemáticas explicadas.

\subsubsection{Demanda Bioquímica de Oxigênio (DBO)}

$$
\frac{\mathrm{dL}}{\mathrm{dt}}=-\mathrm{K}_{\mathrm{d}} \mathrm{L}
$$

$\mathrm{K}_{\mathrm{d}}$ é a constante de desoxigenação em dia $^{-1}$, e L representa a demanda bioquímica de oxigênio em mg/L. A carga pontual lançada na origem do trecho a ser estudado é dada por essa equação. Ao integrarmos a equação diferencial 2.1, chega-se ao seguinte resultado:

$$
\mathrm{L}=\mathrm{L}_{1} \cdot \mathrm{e}^{-\mathrm{K}_{\mathrm{d}} \cdot \mathrm{t}}
$$


A partir das tabelas do estudo de Von Sperling (2007) determinou-se o valor de $\mathrm{K}_{\mathrm{d}}=0,50 \mathrm{dia}^{-1}$. Foi levado em consideração que o rio adotado é raso e recebe esgoto bruto concentrado, de acordo com as especificações estabelecidas pelo autor.

\subsubsection{Déficit de Oxigênio Dissolvido (Déficit de OD)}

A seguinte equação descrita abaixo (2.3), é uma representação matemática do déficit de OD para um lançamento pontual no corpo hídrico.

$\frac{\mathrm{dD}}{\mathrm{dt}}=\mathrm{K}_{\mathrm{d}} \mathrm{L}-\mathrm{K}_{2} \mathrm{D}$

D é o Déficit de OD em $\mathrm{mg} / \mathrm{L}$;

$\mathrm{K}_{2}$ é a constante de reoxigenação do rio em dia $^{-1}$.

Para a determinação de $\mathrm{K}_{2}$ será utilizada a tabela a seguir:

Tabela 1: Valores típicos de $\mathrm{K}_{2}$ (na temperatura de $20^{\circ} \mathrm{C}$ ).

\begin{tabular}{l|c|c}
\hline \multirow{2}{*}{ Corpo d'água } & \multicolumn{2}{c}{$\mathbf{K}_{\mathbf{2}}\left(\mathbf{d i a}^{-1}\right)$} \\
\cline { 2 - 3 } & Profundo & Raso \\
\hline Pequenas lagoas & 0,12 & 0,23 \\
\hline Rios vagarosos, grandes lagos & 0,23 & 0,37 \\
\hline Grandes rios com baixas velocidades & 0,37 & 0,46 \\
\hline Grandes rios com velocidade normal & 0,46 & 0,69 \\
\hline Rios rápidos & 0,69 & 1,15 \\
\hline Corredeiras e quedas d'água & $>1,15$ & $>1,61$ \\
\hline
\end{tabular}

Fonte: Adaptado de Von Sperling (2007).

Pela Tabela 1, o valor de $\mathrm{K}_{2}$ adotado foi 0,46 , sendo considerado o rio em estudo como raso, grande e com baixas velocidades.

Integrando a equação 2.3 chegamos à seguinte expressão:

$D_{t}=\frac{K_{d} L_{0}}{K_{2}-K_{d}}\left(e^{-K_{d} t}-e^{-K_{2} t}\right)+D_{0} e^{-K_{2} t}$

$\mathrm{D}_{\mathrm{t}}[\mathrm{mg} / \mathrm{L}]$ é o Déficit de Oxigênio dissolvido na água em um determinado tempo t.

\subsubsection{Oxigênio dissolvido (OD)}

Como explicado anteriormente, o oxigênio dissolvido é calculado pela diferença entre o oxigênio saturado no corpo hídrico e o déficit de oxigênio dissolvido no rio.

$$
\mathrm{C}_{\mathrm{t}}=\mathrm{C}_{\mathrm{s}}-\mathrm{D}_{\mathrm{t}}
$$

$\mathrm{C}_{\mathrm{s}}$ é a concentração de saturação do oxigênio dissolvido em $\mathrm{mg} / \mathrm{L}$;

$\mathrm{C}_{\mathrm{t}}$ é a concentração do oxigênio dissolvido em um determinado tempo, sendo essa concentração também em mg/L.

Portanto, se substituirmos a equação anterior na equação do déficit (equação 2.3), é obtida a seguinte equação diferencial:

$$
\frac{\mathrm{dC}}{\mathrm{dt}}=-\mathrm{K}_{\mathrm{d}} \mathrm{L}+\mathrm{K}_{2}\left(\mathrm{C}_{\mathrm{s}}-\mathrm{C}_{\mathrm{t}}\right)
$$

A fórmula do oxigênio dissolvido, assim como as outras (DBO e déficit de OD), pode ser integrada a fim de se conseguir resolver a equação diferencial, chegando-se à seguinte equação: 


$$
C_{t}=C_{s}-\left\{\frac{K_{d} L_{0}}{K_{2}-K_{d}}\left(e^{-K_{d} t}-e^{-K_{2} t}\right)+\left(C_{s}-C_{t}\right) e^{-K_{2} t}\right\}
$$

Segundo Thomann (1989), para calcularmos a concentração de saturação do oxigênio dissolvido $\left(\mathrm{C}_{\mathrm{s}}\right)$ deve-se partir da seguinte expressão:

$$
C_{s}=\exp (z)
$$

Sendo z obtido a partir da seguinte equação:

$$
z=-139,34411+\frac{1,575701 * 10^{5}}{T}-\frac{6,642308 * 10^{7}}{T^{2}}+\frac{1,2438 * 10^{10}}{T^{3}}-\frac{8,621949 \cdot 10^{11}}{T^{4}}
$$

T é a temperatura da água em Kelvin.

\subsection{Análise de déficit e concentração de $O D$}

Neste trabalho, foram analisadas, para o processo de autodepuração do rio Piranhas-Açu, o déficit de oxigênio dissolvido e as concentrações do oxigênio dissolvido ao longo do trecho determinado. A partir do lançamento do efluente na origem de um determinado trecho do rio Piranhas-Açu, foi simulado para cada $100 \mathrm{~m}$ o déficit de OD e sua concentração de OD ao longo de aproximadamente $54,5 \mathrm{~km}$. Dessa forma, tem-se mais de 500 resultados para o déficit e a concentração.

\subsection{Caracterização da região}

Para o presente trabalho foi estudado o rio Piranhas-Açu, localizado no estado da Paraíba e Rio Grande do Norte, tendo sua nascente na junção dos rios do Peixe e Piancó, na Paraíba. É conhecido como Piranhas até passar a barragem Armando Ribeiro Gonçalves, no município de Itajá. Já no Rio Grande do Norte recebe o nome de Açu e deságua na cidade de Macau. Com aproximadamente $400 \mathrm{~km}$ de comprimento, possui a maior bacia, tanto do Rio Grande do Norte quanto no estado da Paraíba, a com em torno de 43.681,5 $\mathrm{Km}^{2}$ de drenagem.

Foi utilizado, para a simulação, um trecho do rio Piranhas-Açu de aproximadamente $55 \mathrm{~km}$ de comprimento. Dentro desse trecho utilizaram-se dados de três seções diferentes coletadas pelo programa de monitoramento e fiscalização da Coordenadoria de Gestão de Recursos Hídricos da Secretaria dos Recursos Hídricos do Estado do Rio Grande do Norte. (Figura 1)

Figura 1 - Trecho escolhido para estudo.

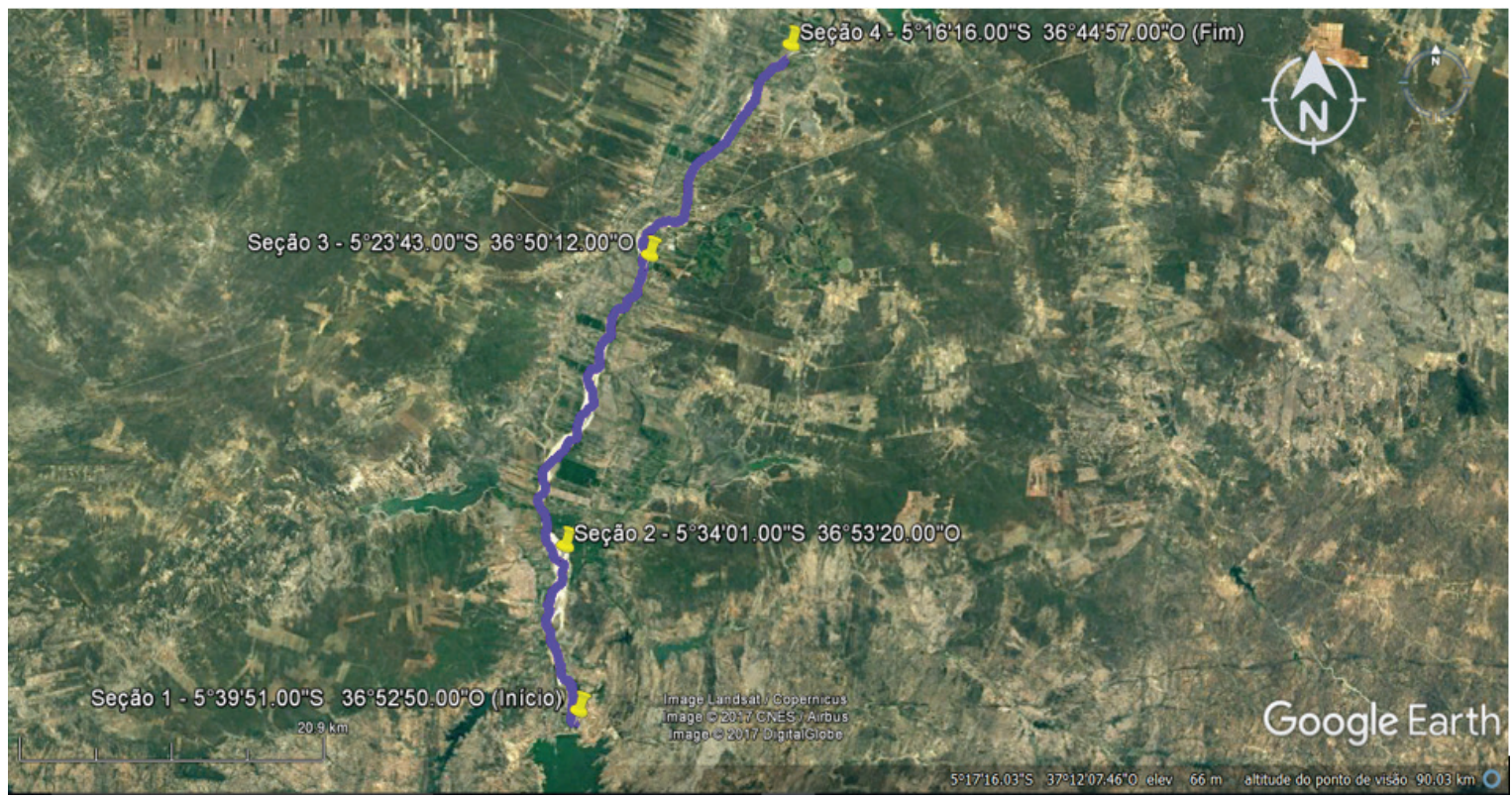

Fonte: Google Earth (2015). 
Os dados das seções utilizadas foram coletados no dia 20 de agosto de 2002 e podem ser encontrados na Tabela 2:

Tabela 2: Dados das seções.

\begin{tabular}{|c|c|c|c|c|c|}
\hline Seção & \multicolumn{2}{|c|}{ Coordenadas } & Área $\left(\mathrm{m}^{2}\right)$ & Velocidade $(\mathrm{m} / \mathrm{s})$ & Velocidade (m/dia) \\
\hline 1 & S 05³9’51” & W $36^{\circ} 52^{\prime} 50^{\prime \prime}$ & 12,68 & 0,79 & 68256 \\
\hline 2 & S $05^{\circ} 34^{\prime} 01^{\prime \prime}$ & W $36^{\circ} 53^{\prime} 20^{\prime \prime}$ & 22,06 & 0,38 & 32832 \\
\hline 3 & $\mathrm{~S} 05^{\circ} 23^{\prime} 43^{\prime \prime}$ & W $36^{\circ} 50^{\prime} 12^{\prime \prime}$ & 14,76 & 0,37 & 31968 \\
\hline 4 & S $05^{\circ} 16^{\prime} 16^{\prime \prime}$ & W $36^{\circ} 44^{\prime} 57^{\prime \prime}$ & ----- & ---- & ---- \\
\hline Seção & \multicolumn{2}{|c|}{ Vazão $\left(\mathbf{m}^{3} / \mathbf{s}\right)$} & o (m) & \multicolumn{2}{|c|}{ Distância acumulada (m) } \\
\hline 1 & \multicolumn{2}{|l|}{9,957} & 0 & \multicolumn{2}{|l|}{0} \\
\hline 2 & \multirow{2}{*}{\multicolumn{2}{|c|}{$\begin{array}{l}8,369 \\
5,524\end{array}$}} & 12065 & \multicolumn{2}{|c|}{12065} \\
\hline 3 & & & & \multirow{2}{*}{\multicolumn{2}{|c|}{$\begin{array}{l}36531 \\
54344\end{array}$}} \\
\hline 4 & \multicolumn{2}{|l|}{----} & 17813 & & \\
\hline
\end{tabular}

Fonte: Autor (2015).

\subsection{Cenários de análise e simulação}

Para a simulação do modelo de Streeter-Phelps, os dados utilizados foram: temperatura, de $26^{\circ} \mathrm{C}$; DBO inicial do rio, com concentração de $2,0 \mathrm{mg} / \mathrm{L}$; vazão do efluente, de $0,1 \mathrm{~m} / 3 / \mathrm{s}$; OD inicial do rio, igual a 7,0 mg/L; e OD do efluente igual, a $0,1 \mathrm{mg} / \mathrm{L}$. Também foram necessários ajustes nos coeficientes da temperatura, para isso foram utilizadas as equações 2.10 e 2.11 .

$$
\begin{aligned}
& \mathrm{K}_{2_{\mathrm{T}}}=\mathrm{K}_{2_{20}} \cdot \theta^{(\mathrm{T}-20)} \\
& \mathrm{K}_{\mathrm{d}_{\mathrm{T}}}=\mathrm{K}_{\mathrm{d}_{20}} \cdot \theta^{(\mathrm{T}-20)}
\end{aligned}
$$

\subsubsection{Cenário 1}

O primeiro cenário consta de uma análise do déficit de OD e da concentração de OD para diferentes concentrações de lançamentos de efluentes na origem do trecho analisado. Sendo assim, foram utilizadas, neste primeiro cenário, cinco concentrações de lançamento de efluentes diferentes, variando de $100 \mathrm{mg} / \mathrm{L}$ a $500 \mathrm{mg} / \mathrm{L}$ para o mesmo trecho sob as condições normais do rio. A partir desses resultados, dois gráficos foram gerados, em que aparecem as cinco curvas de dispersão, tanto para o déficit de OD como para a concentração do OD.

\subsubsection{Cenário 2}

Para o segundo cenário, foram analisados os mesmos parâmetros do cenário 1, déficit e concentração de oxigênio dissolvido, porém foram verificadas as condições do rio dentro dos limites estabelecidos pelo Conselho Nacional do Meio Ambiente - CONAMA, por meio da Resolução no 375 de 2005. Com os dados coletados no primeiro cenário, foram adicionados aos gráficos os limites estabelecidos pela resolução e, assim, analisadas as condições do rio perante tal resolução para as diferentes concentrações de efluentes a serem lançadas no corpo hídrico.

\subsubsection{Cenário 3}

No terceiro e último cenário, foi estudado o caso durante o período de seca e de cheia para o rio Piranhas-Açu. Para tal fim, foram estabelecidas duas vazões fixas para todo o trecho do rio, sendo uma vazão para o período de seca e outra para o período de cheia.

Essas vazões foram definidas a partir de um estudo realizado por Chagas (2005) sobre o rio Potengi, que fica localizado no estado do Rio Grande do Norte. Por ser um rio próximo, com características semelhantes, e pela falta de dados sobre a vazão do rio Piranhas-Açu no período de seca ou cheia, optou-se pela escolha do rio Potengi quanto às vazões utilizadas para o terceiro cenário. Sendo essas vazões de $14,5 \mathrm{~m}^{3} / \mathrm{s}$ para o período de cheia e de $2,6 \mathrm{~m}^{3} / \mathrm{s}$ para a simulação do período de seca. 
Foram adotadas também novas áreas para as seções, considerando-as como sendo todas iguais em toda a extensão do trecho. Foi utilizada, para o período de cheia, a média das áreas utilizadas nas simulações anteriores, $16,95 \mathrm{~m}^{2}$; e, para o período de estiagem, foi usada uma proporção entre as vazões para encontrar uma área igualmente proporcional para o mesmo período, sendo essa área igual a $2,96 \mathrm{~m}^{2}$.

Novamente, foram simulados os déficits e as concentrações de OD para as cinco diferentes concentrações de lançamentos de efluentes utilizadas no primeiro cenário. Com isso, foram gerados gráficos de cheia e seca para os dois parâmetros estudados nos cenários anteriores. Sendo, portanto, analisadas as condições do rio nos dois períodos, como ele se comporta mediante o lançamento de diferentes concentrações de efluentes e sua capacidade de autodepuração.

\section{Resultados e discussões}

Aplicando ao modelo os dados apresentados anteriormente, foram elaborados gráficos para o déficit e para o oxigênio dissolvido nos diversos cenários e nas diferentes concentrações de efluentes. Através dos gráficos construídos podemos ver como o rio se comporta para diferentes concentrações de efluentes e comparar a autodepuração do rio em épocas de estiagem e de cheia, além de checar se, para diferentes classes de uso, a concentração de oxigênio dissolvido no corpo hídrico fica dentro dos limites estabelecidos pelo CONAMA na Resolução n ${ }^{0}$ 357/2005. Os resultados são mostrados e analisados a seguir.

\subsection{Cenário 1}

O primeiro cenário apresenta a análise do déficit e também da concentração de oxigênio dissolvido, no trecho indicado do rio, para diferentes concentrações de efluentes sendo lançados no ponto de origem do trecho. Logo, na primeira simulação, ilustrada na Fig. 2 a seguir, verifica-se o comportamento das curvas de concentração de OD ao longo do trecho para diferentes concentrações de efluentes de uma fonte pontual no início do trecho.

Figura 2 - Concentração de OD para diferentes concentrações de efluentes.

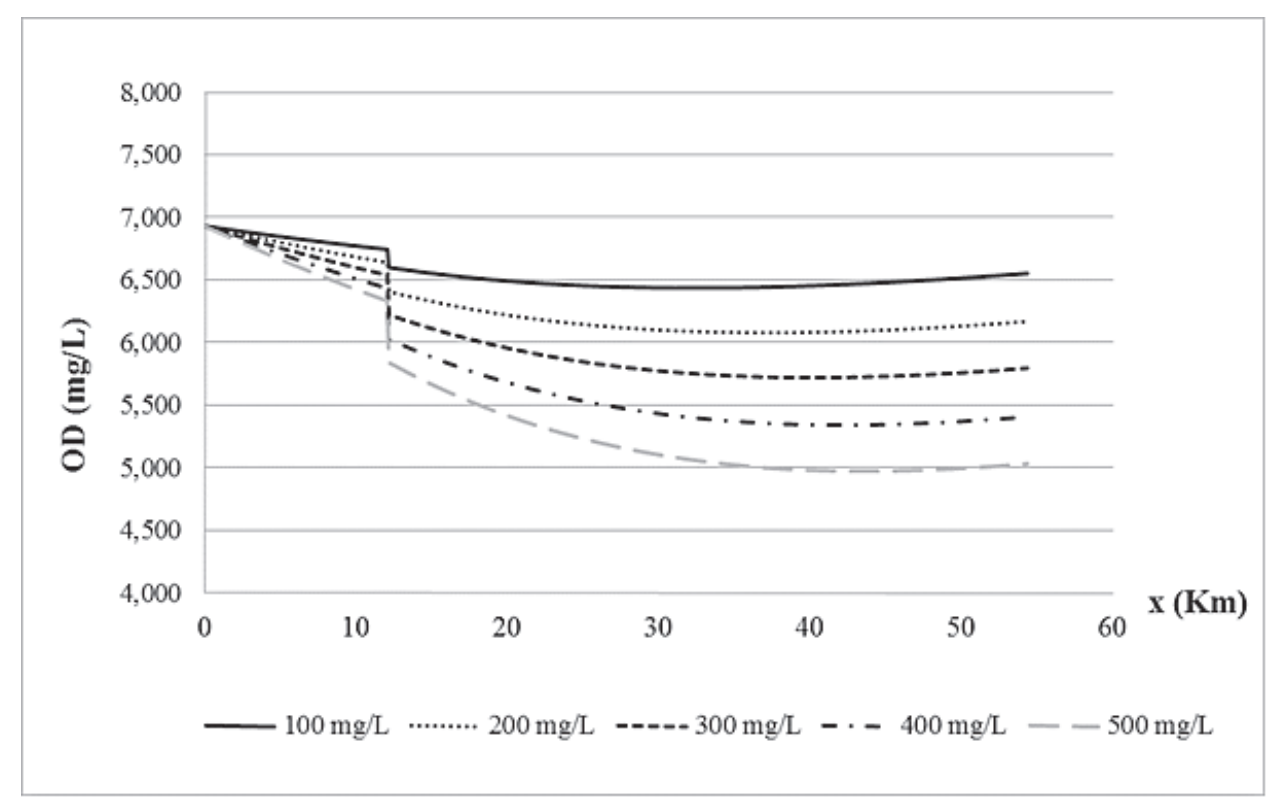

Na Figura 2, podemos ver o decaimento da concentração do oxigênio dissolvido a partir do lançamento do efluente no início do trecho e, próximo aos $30 \mathrm{~km}$, para um lançamento de $100 \mathrm{mg} / \mathrm{L}$, o aumento das concentrações de OD, o que significa que o corpo hídrico está começando a se "recuperar" da carga de efluente sobre ele lançada. Próximo aos $12 \mathrm{~km}$ é visível uma queda brusca na concentração de OD do rio. Essa queda ocorre no ponto de mudança entre as duas primeiras seções, pois há uma grande diferença na velocidade da água no rio, o que acaba provocando esse rebaixamento de OD no gráfico da Figura 2. Da mesma forma, vemos o ocorrido na análise da Fig. 3, que representa o déficit de OD no mesmo trecho. Podemos ver como o déficit se comporta de maneira exatamente oposta à concentração de OD, com 
um claro aumento inicial no déficit e uma leve diminuição nos quilômetros seguintes. Para a concentração de $100 \mathrm{mg} / \mathrm{L}$, verifica-se que o ponto mais alto da curva do déficit, e por consequência o mais baixo da concentração de OD, ocorre perto dos $32 \mathrm{Km}$. Agora, para o lançamento de $200 \mathrm{mg} / \mathrm{L}$, esse ponto de déficit máximo e de concentração mínima de OD ocorre um pouco depois, já próximo dos $37 \mathrm{Km}$ do início do trecho, como esperado. Conforme a concentração do efluente aumenta, essa distância também aumenta gradativamente, chegando em $40 \mathrm{Km}$ para uma concentração de 300 mg/L de efluente; $43 \mathrm{Km}$ para $400 \mathrm{mg} / \mathrm{L}$ e $45 \mathrm{Km}$ para a maior concentração de efluente lançado estudado, de $500 \mathrm{mg} / \mathrm{L}$. A partir desse ponto, tem-se uma diminuição do déficit e, consequentemente, um aumento para a concentração de OD.

Figura 3 - Déficit de OD Para diferentes concentrações de efluentes.

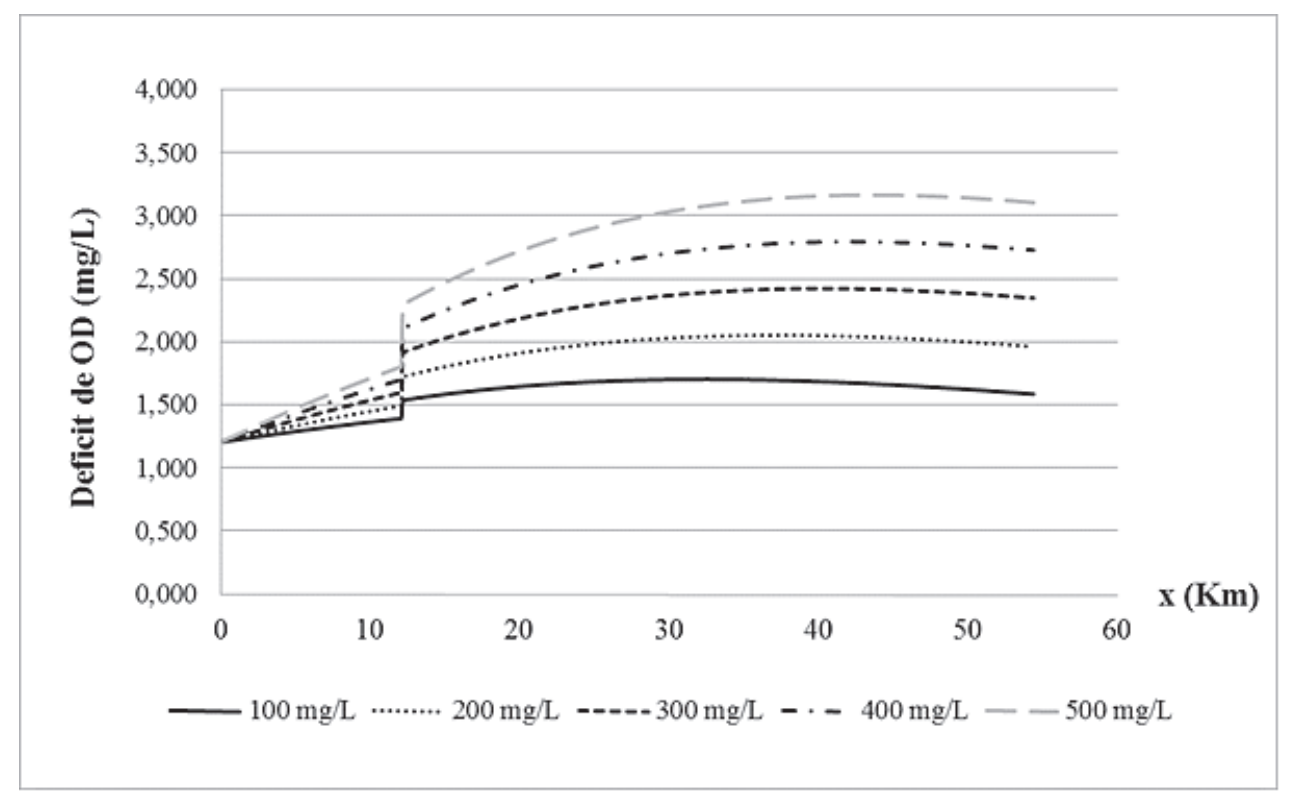

Esses níveis de OD e de déficit tendem a se normalizarem ao longo do curso do rio se não ocorrerem mudanças ou interferências, como mais lançamentos de efluentes. A normalização do déficit e da concentração de OD tende a ocorrer de forma cada vez mais lenta.Quanto mais próximo do nível normal eles estiverem, o déficit vai tender a zero, enquanto a concentração de OD tenderá para seu valor máximo, que é o valor de saturação do oxigênio para a água em temperatura ambiente.

\subsection{Cenário 2}

No segundo cenário simulado para o rio Piranhas-Açu, temos as mesmas concentrações de efluentes sob as mesmas condições do primeiro cenário. Porém, nesse caso, não foi analisada a capacidade de autodepuração do rio, uma vez que já foi verificada no cenário anterior, sendo analisado se as concentrações de oxigênio dissolvido na água do rio estão dentro dos parâmetros estabelecidos pela Resolução no 357/2005 do CONAMA.

O rio Piranhas-Açu não está classificado pelo CONAMA. No capitulo VI da Resolução n 357/2005 do Conselho Nacional do Meio Ambiente, o artigo 42 estabelece que, enquanto não aprovados os respectivos enquadramentos, as águas doces serão consideradas de classe 2. Portanto, para esta pesquisa, o rio Piranhas-Açu é considerado um rio de classe 2 para os limites de concentração de OD estabelecidos na referida resolução.

Nesse cenário, foram consideradas todas as classes de rios, apesar de já enquadrar o rio foco da pesquisa em classe 2. É possível, portanto, analisar os limites de todas as diferentes classes e como o rio Piranhas-Açu se comportaria caso estivesse classificado em cada uma delas. É válido lembrar que a resolução determina que as águas de classe especial devem ser mantidas em suas condições naturais. Por esse motivo, essa classe não foi simulada.

A Figura 4 ilustra as concentrações de OD para as diferentes concentrações de lançamentos de efluentes e o limite da concentração de OD para rios de classe 1. Observa-se que três dos cinco lançamentos simulados para o rio acabam por fazer com que as concentrações de oxigênio dissolvido no corpo hídrico sejam inferiores ao limite estabelecido para a 
classe 1. Portanto, se o rio Piranhas-Açu fosse classificado como sendo um rio de classe 1, não poderia receber efluentes de concentrações maiores do que $200 \mathrm{mg} / \mathrm{L}$ nas condições simuladas.

Figura 4 - Concentração de OD com limites para rios de classe 1.

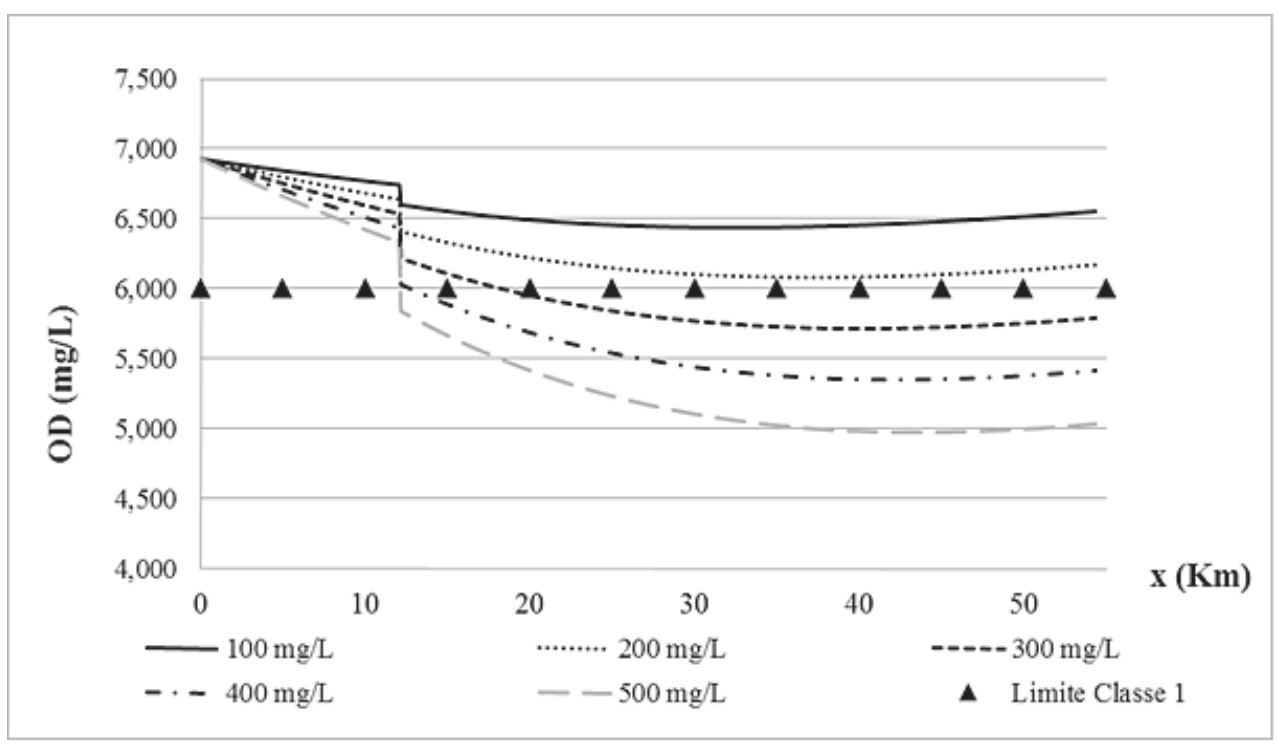

Conforme mostra a Fig. 5, constam as mesmas concentrações e parâmetros utilizados anteriormente para a concentração de OD no trecho escolhido do rio, considerado como classe 2 segundo o CONAMA. É possível visualizar no gráfico que todas as cinco diferentes concentrações de efluentes simuladas para o trecho estão dentro dos parâmetros exigidos pela resolução e para rios de classe 2 . O menor valor de concentração de OD que temos é na curva de $500 \mathrm{mg} / \mathrm{L}$, como esperado, próximo à distância de $46 \mathrm{Km}$ depois do lançamento do efluente. Nesse ponto, a concentração de OD atinge o seu menor valor, correspondendo a $4,971 \mathrm{mg} / \mathrm{L}$.

Como verificado na Fig. 5, pode-se constatar que esse rio é passível de receber lançamentos de até $500 \mathrm{mg} / \mathrm{L}$ nos trechos estudados, conforme os padrões estabelecidos na Resolução n ${ }^{\circ}$ 357/2005 do CONAMA para o parâmetro de concentração de OD.

Figura 5 - Concentração de OD com limites para rios de classe 2.

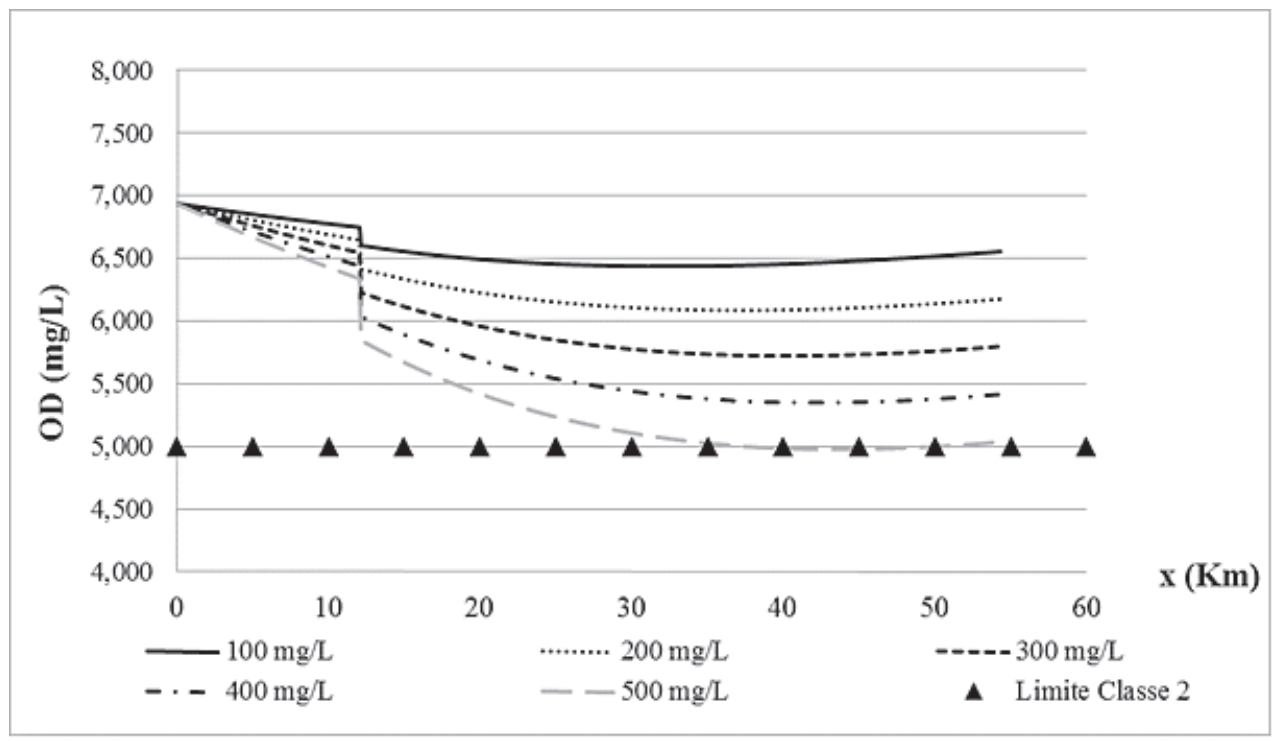


Os limites de concentração de OD para as classes diminuem conforme o número da classe aumenta, ou seja, o limite para a classe 1 é maior do que o para a classe 2, e o de classe 2 é maior do que o limite para a classe 3, e assim por diante. Por esse motivo, pode-se assumir que, para as condições estabelecidas nesta pesquisa, bem como para as mesmas concentrações de efluentes utilizadas, o rio pode ser outorgado para lançamentos de até $400 \mathrm{mg} / \mathrm{L}$ se levarmos em conta os limites de OD, o que vale para a classificação do rio em classe 2. Para as classes 3 ou 4, o rio pode ser outorgado para lançamentos de até $500 \mathrm{mg} / \mathrm{L}$, como podemos conferir nas Figuras 6 e 7.

Figura 6 - Concentração de OD com limites para rios de classe 3.

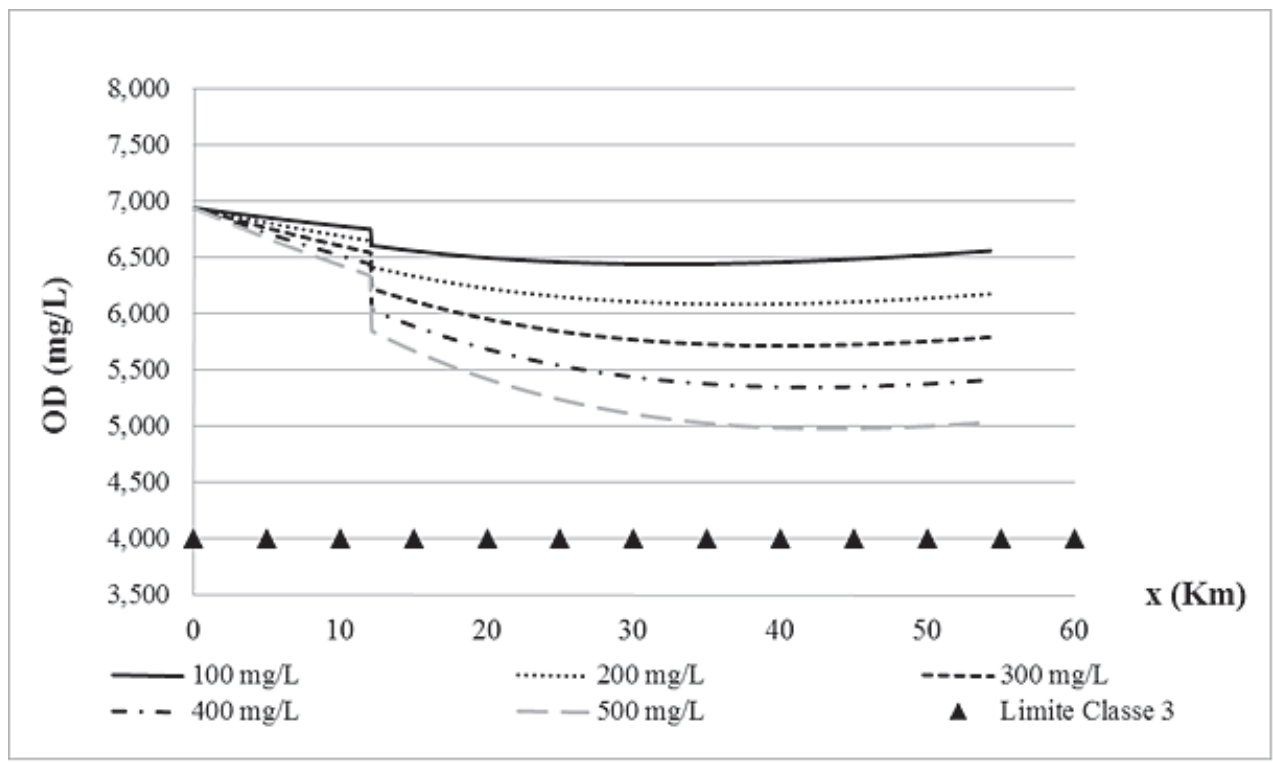

Figura 7 - Concentração de OD com limites para rios de classe 4.

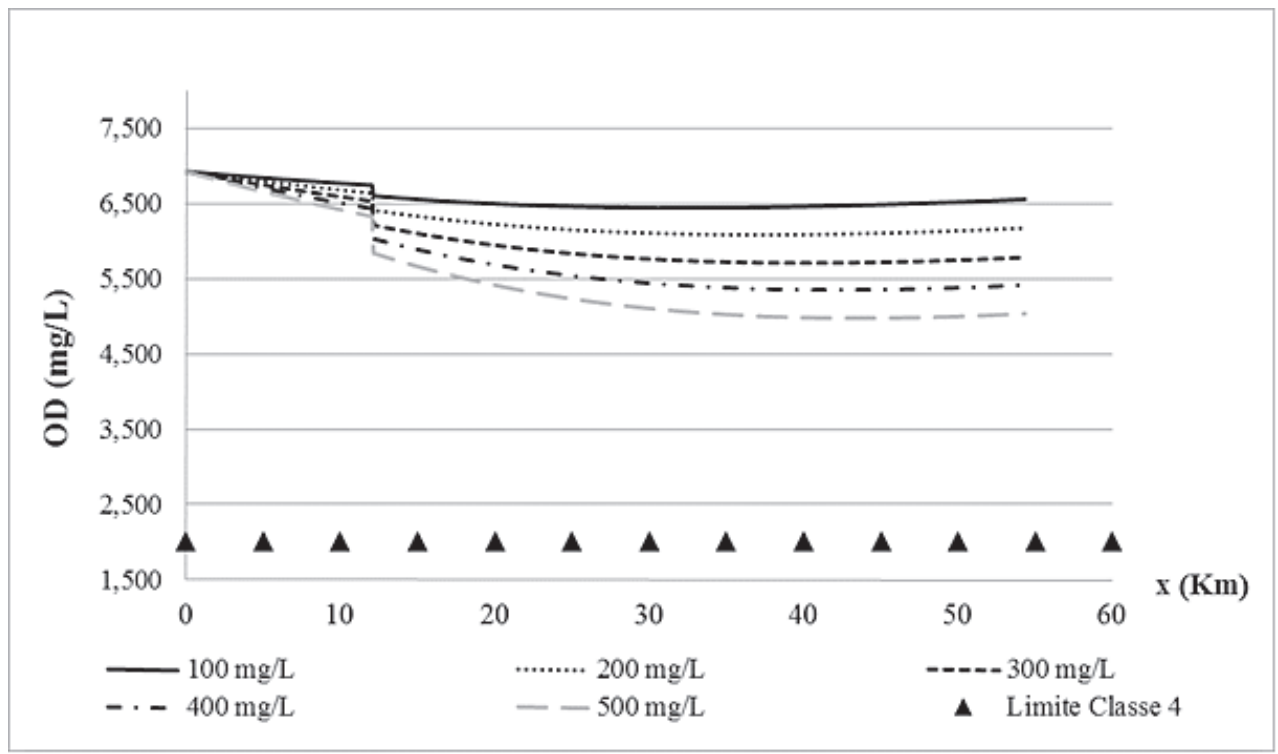




\section{3 Cenário 3}

Para o terceiro e último cenário, foi simulado o comportamento do rio em relação à sua concentração de OD, em períodos de seca e cheia. Para tanto, foram estabelecidas as vazões em cada período e fixada a vazão para todo o trecho, mudando apenas a velocidade das águas de acordo com a área da seção, utilizando como parâmetro a classificação do rio segundo o CONAMA, que o classifica como de classe 2.

São apresentadas na Fig. 8 as concentrações de OD do rio Piranhas-Açu em um período de cheia, para diferentes concentrações de efluentes simuladas, considerando uma fonte pontual lançada no início do trecho.

Figura 8 - Concentrações de OD para período de cheia

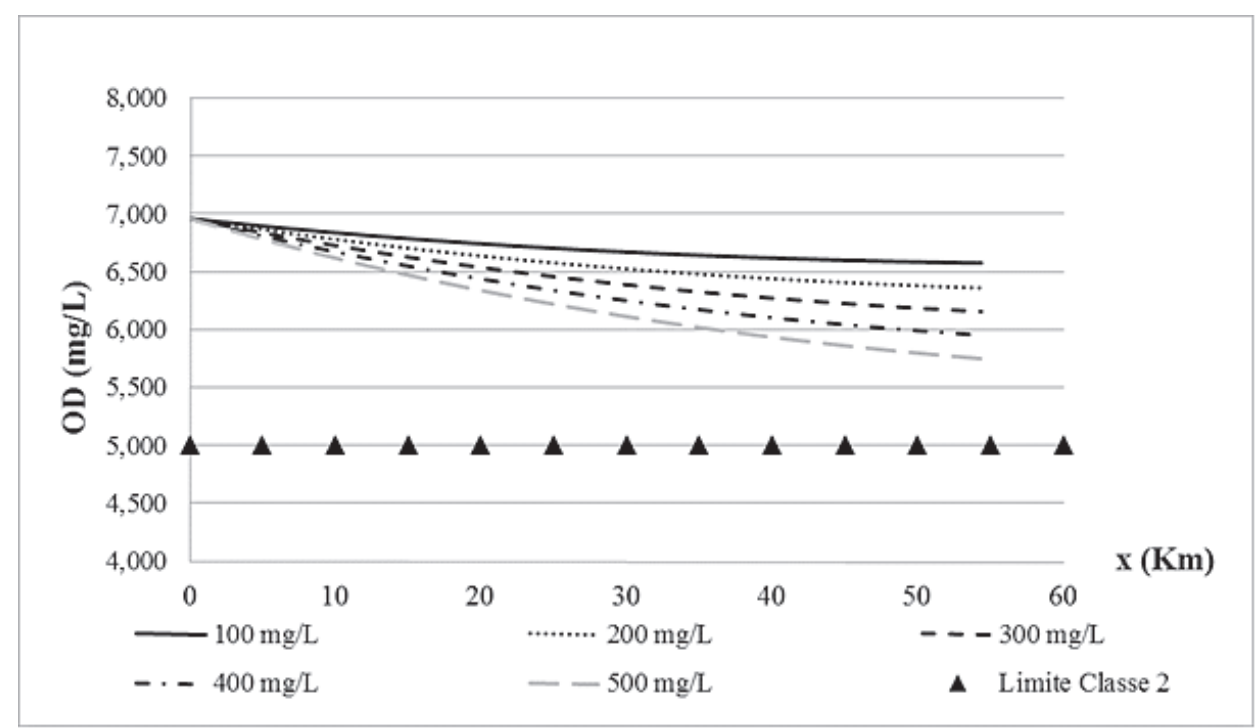

Na Figura 8 é ilustrada uma queda da concentração de OD de forma lenta, pois como a vazão aumenta e foi utilizada uma área média para o rio, temos uma velocidade constante e alta em todo o percurso, fazendo com que a curva seja suave e a recuperação do rio só venha a ocorrer em pontos mais distantes do início do trecho.

A Figura 9 ilustra a concentração de OD do rio Piranhas-Açu para um suposto período de seca, com as diferentes concentrações de efluentes simuladas, considerando uma fonte pontual no início do trecho.

Figura 9 - Concentrações de OD para período de estiagem.

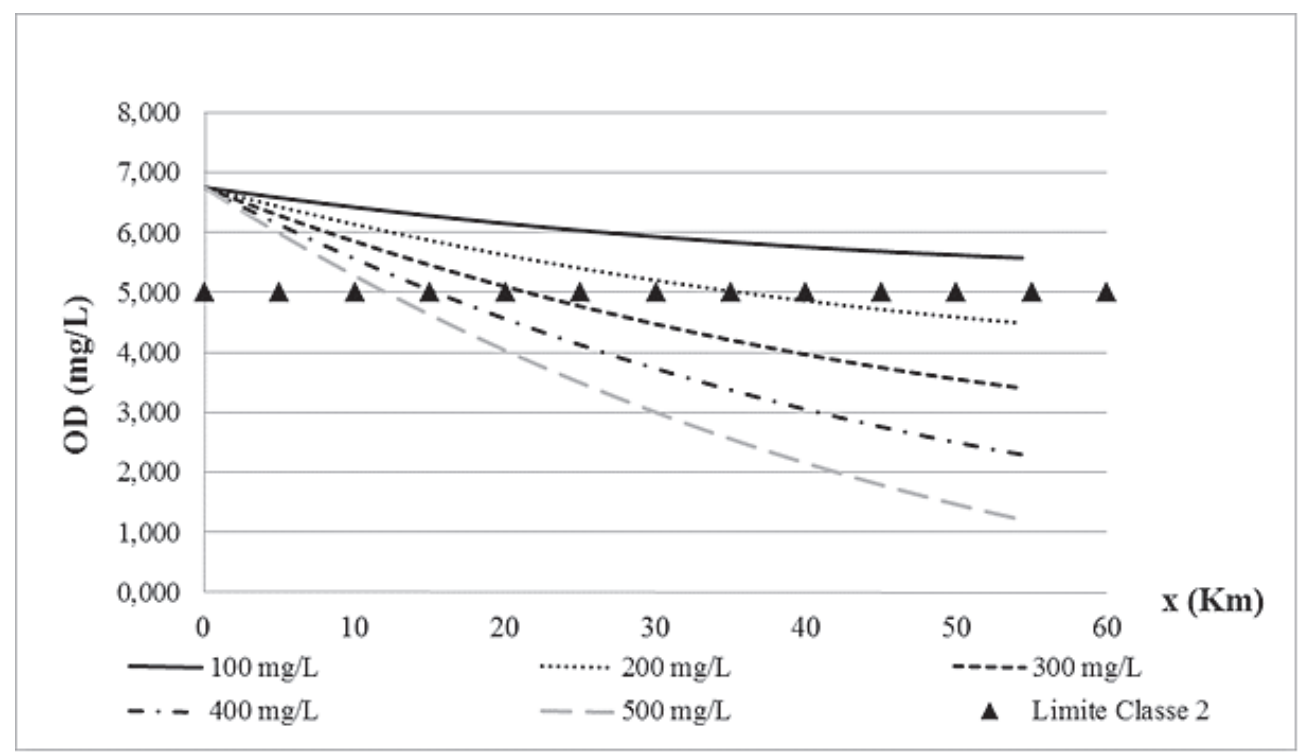


Devido à baixa vazão e à pequena área adotada para o período de seca, verificam-se baixas velocidades nas águas do rio, o que faz com que o processo de recuperação do rio se torne mais lento e menos efetivo. No período de seca, é visível a diminuição de sua capacidade de autodepuração, assim como se percebe que o rio não suporta lançamentos de efluentes muito maiores do que $100 \mathrm{mg} / \mathrm{L}$, limite estabelecido pelo CONAMA para rios de classe 2 .

\section{Conclusão e recomendações}

Os resultados gerados para as simulações de diferentes concentrações de efluentes lançadas no trecho do rio PiranhasAçu sobre diversos cenários possibilitaram estabelecer a seguintes conclusões:

No primeiro cenário, observou-se como se comporta o OD e o déficit de OD no rio Piranhas-Açu para os lançamentos simulados. Observou-se que a concentração de OD passa a aumentar em diferentes pontos do trecho para cada concentração de efluente simulada. Quanto maior a concentração de efluente, maior será o tempo de autodepuração do rio.

Para o segundo cenário, constatou-se que o rio Piranhas-Açu, caso seja considerado um rio de classe 1, pode ser outorgado para lançamentos de até $200 \mathrm{mg} / \mathrm{L}$. Caso classificado como classe 2, pode ser outorgado para lançamentos de até $400 \mathrm{mg} / \mathrm{L}$, e, caso seja considerado um rio de classe 3 ou 4, pode ser outorgado lançamentos maiores do que $500 \mathrm{mg} / \mathrm{L}$.

No terceiro cenário foram ilustradas as concentrações para períodos simulados de cheia e de seca, em que foi constatado que, com o aumento da vazão no rio no período de cheia, a capacidade de autodepuração do rio também aumenta, fazendo com o que ele possa ser outorgado para lançamentos de até $500 \mathrm{mg} / \mathrm{L}$ nos períodos de cheia. Já nos períodos de seca, com a diminuição da vazão, a capacidade de autodepuração do rio é reduzida, de maneira que o rio suporta apenas, para a classificação de rio classe 2 , lançamentos de até $100 \mathrm{mg} / \mathrm{L}$.

Por fim, este tipo de estudo facilita o estabelecimento de critérios de avaliação de qualidade de água, consequentemente a tomada de decisão por parte de gestores ambientais sobretudo em locais carentes de informação, uma vez que não necessita de muitos dados de entrada para um diagnóstico preliminar.

\section{Referências}

ALVAREZ, R. P. P. Estudo experimental e teórico da qualidade de água da drenagem urbana com base ecohidrológica. 2010. 290f. Dissertação (Mestrado em Hidráulica e Saneamento) - Escola de Engenharia de São Carlos, Universidade de São Paulo, São Carlos, 2010.

BRASIL. Resolução $\mathrm{n}^{\circ}$. 430, de 13 de maio de 2011. Dispõe sobre as condições e padrões de lançamento de efluentes, complementa e altera a Resolução no 357, de 17 de março de 2005. Diário Oficial da União, Brasília, DF. Disponível em: <http://www.mma.gov.br/port/conama/legiabre.cfm?codlegiHYPERLINK "http://www.mma.gov.br/port/conama/ legiabre.cfm?codlegi $=646 "=646>$. Acesso em: 8 jan. 2016.

. Resolução n 357 , de 17 de março de 2005. Dispõe sobre a classificação dos corpos de água e diretrizes ambientais para o seu enquadramento, bem como estabelece as condições e padrões de lançamento de efluentes, e dá outras providências, de 17 de março de 2005. Diário Oficial da União, Brasília, DF, 18 mar. 2005, p. 58-63.

CHAGAS, P. F. Perspectivas da aplicação da teoria fuzzy para cálculo de risco em sistemas hidrodinâmicos. 2005. 189 f. Tese (Doutorado), Universidade Federal do Ceará, Fortaleza, 2005.

CHAPRA S. C. Surface water-quality modeling. New York: McGraw-Hill, 1997. (McGraw-Hill series in water resources and environmental engineering).

DOBBINS W. E. Bod and oxygen relationship in streams. Journal of the Sanitary Engineering Division. New York, v. 90, n. 3 p. $53-78,1964$.

HU, B. New strategies for environmental water analysis. In: INTERNATIONAL CONFERENCE AND EXHIBITION ON WATER AND THE ENVIRONMENT, 2009, Stellenbosch. Proceedings... Stellenbosch: 2009. 1 CD-ROM

O'CONNOR D. J. The temporal and spatial distribution of dissolved oxygen in streams. Water Resources Research. Florida, v. 3, n. 1, p. 65-79, 1967

SALES, R. J. M. Aplicação da lógica Fuzzy no modelo de Streeter-Phelps para analisar o risco de contaminação das águas de rios, considerando múltiplos processos e múltiplos lançamentos. 2014. 116 f. Tese (Doutorado em Engenharia Civil) - Universidade Federal do Ceará, Fortaleza, 2014. 
SANDERS, A. A. Desenvolvimento de uma metodologia, com base na teoria Fuzzy, aplicada a modelos da demanda bioquímica de oxigênio (DBO) e oxigênio dissolvido (OD), para calcular o risco de degradação ambiental em rios naturais. 2009. 140 f. Tese (Doutorado em Engenharia Civil) - Universidade Federal do Ceará, Fortaleza, 2009.

SPERLING V. M. Estudos e modelagem da qualidade da água de rios. Belo Horizonte: UFMG, 2007.

V. M. Introdução à qualidade das águas e ao tratamento de esgotos. 3. ed. Belo Horizonte: UFMG, 2005.

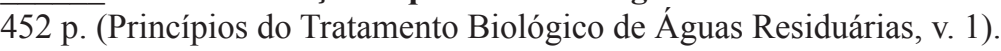

STREETER H. W.; PHELPS E. B. A study of the pollution and natural purification of the Ohio River. III. Factors concerning the phenomena of oxidation and reaeration. Pub. Health Bulletin._n. 46, 1958.

THOMANN R.V. Bioaccumulation model of organic chemical distribution in aquatic food chains. Environ. Sci. Techno., Washington, v. 18, p. 67-71, 1989.

VASCONCELLOS, K. C. Estudo do risco de falha ambiental em rios sujeitos a concessão de outorga de lançamentos de efluentes, mediante o uso da equação de Streeter-Phelps "fuzzificada". 2013. 74 f. Dissertação (Mestrado em Engenharia Civil) - Universidade Federal do Ceará, Fortaleza, 2013.

Sobre os autores

\section{Victor Prisco Diogo de Holanda}

Engenheiro Civil, Universidade de Fortaleza - UNIFOR.

\section{Thainá Rayanne Soares}

Estudante de Engenharia Civil, Universidade de Fortaleza - UNIFOR.

\section{Roani Simões Veras}

Engenheiro Civil, Universidade de Fortaleza - UNIFOR.

\section{Ingrid Fernandes de Oliveira Alencar}

Estudante de Engenharia Civil, Universidade de Fortaleza - UNIFOR.

\section{Raquel Jucá de Moraes Sales}

Tecnóloga em Saneamento Ambiental - Instituto Federal de Educação, Ciência e Tecnologia do Ceará, M.Sc e Doutora em Engenharia Civil - Recursos Hídricos - Universidade Federal do Ceará. Professora Auxiliar do Centro de Ciências Tecnológicas da Universidade de Fortaleza. 